\title{
Inversor de tensão com topologia push-pull para injeção de energia em sistemas elétricos com modulação SPWM
}

\section{Voltage inverter with push-pull topology to inject energy into electrical systems with modulation SPWM}

\author{
Emerson Charles M. Silva ${ }^{1}$; Carlos Henrique G. Treviso²;
}

\begin{abstract}
Resumo
Este trabalho apresenta uma proposta de um inversor de tensão baseado na topologia de conversores push-pull, chaveado em alta freqüência para injeção de energia na rede elétrica a partir de uma fonte de energia em corrente contínua. Um sistema de inversão de tensão utilizando dois conversores estáticos fornece à rede elétrica, energia em forma de corrente alternada, que pode trabalhar em conjunto com o fornecimento da concessionária de energia. O projeto visa o aproveitamento de possíveis fontes de energia renováveis que possam ser armazenadas em forma de tensão contínua, tais como, eólica, solar, hidroelétrica entre outras. O funcionamento da topologia é apresentado, tal como os circuitos de potência e controle, assim como dimensionamento de componentes, resultados teóricos e práticos conseguidos com a montagem de um protótipo de $100 \mathrm{~W}$ de potência e chaveamento em $40 \mathrm{khz}$, que após filtragem fornece a frequência de $60 \mathrm{~Hz}$ que é compatível com o sistema elétrico brasileiro.
\end{abstract}

Palavras-chave: Inversor de tensão. Push-pull. SPWM. Injeção de energia.

\begin{abstract}
This paper presents a proposal for a voltage inverter topology based on push-pull converters, switched at high frequency to inject energy into the grid from a source of DC power. A system using two reverse voltage static converters provides the power grid; energy in the form of alternating current, that can work in conjunction with the provision of utility power. Aiming at the possible use of renewable energy sources, that can be stored in the form of voltage continuous, such as wind, solar, hydroelectric and others. The functioning of topology is presented, such as the power and control circuits, as well as sizing components, theoretical and practical results achieved with the assembly of a prototype $100 \mathrm{~W}$ of power and switching in $40 \mathrm{khz}$, which after filtering provides the frequency of $60 \mathrm{~Hz}$, which is compatible with the Brazilian electrical system.
\end{abstract}

Keywords: Voltage inverter. Push-pull. SPWM. Injection energy.

\footnotetext{
${ }^{1}$ Docente do Departamento de Engenharia de Controle e Automação do Centro Universitário de Maringá; emertins@hotmail.com.

${ }^{2}$ Docente do Departamento de Engenharia Elétrica da Universidade Estadual de Londrina-DEEL-UEL; treviso@uel.br.
} 


\section{Introdução}

O inversor de tensão desenvolvido utiliza modulação SPWM (RODRIGUES e TREVISO 2007), e a técnica de chaveamento conhecida como classe D (BOYLESTAD; NASHELSKY, 2011), e aqui se apresenta para a utilização em um sistema de injeção de energia elétrica em forma de corrente na rede elétrica. O inversor de potência foi baseado nos conversores PUSH_PULL (MELLO, 2011 e BARBI, 2001) onde são utilizados dois conversores, um trabalhando no semi-ciclo positivo e outro no semi-ciclo negativo no modo de condução contínua de corrente. É possível elevar a tensão e obter a inversão de uma tensão de $12 \mathrm{Vcc}$ para uma tensão de $127 \mathrm{Vrms}$, que com a técnica apresentada pode ter sua potência inserida em conjunto com a rede elétrica da concessionária. Para a geração dos pulsos das chaves dos conversores, é utilizada uma amostra do sinal senoidal da rede na comparação com uma onda triangular, ou seja, o sistema deverá injetar potência na rede, sincronizado com o sinal da mesma, e apenas quando houver tensão da concessionária.

Uma ação integral (BARBI; MARTINS, 2005) é efetuada entre o sinal de tensão da rede elétrica e o sinal de corrente na carga, o resultado é comparado com uma onda triangular para geração do sinal pwm, o que permite uma ação de controle na saída do inversor. O diagrama de blocos do inversor é apresentado na figura 1 que enfatiza o sinal de saída sendo amostrado na entrada para a formação do sinal de acionamento das chaves, que é desmembrado no circuito de controle separando os pulsos que atuarão no semi-ciclo positivo, e no semi-ciclo negativo. $\mathrm{O}$ circuito secundário é controlado por duas chaves bidirecionais, desenvolvidas com transistores e diodos, que controla a direção da corrente na carga de acordo com a sequência de atuação dos conversores, assim, pode-se injetar a energia dos conversores na rede para obter corrente nos dois sentidos, fornecendo à rede, a corrente alternada.
Figura 1 - Diagrama de blocos do conversor.

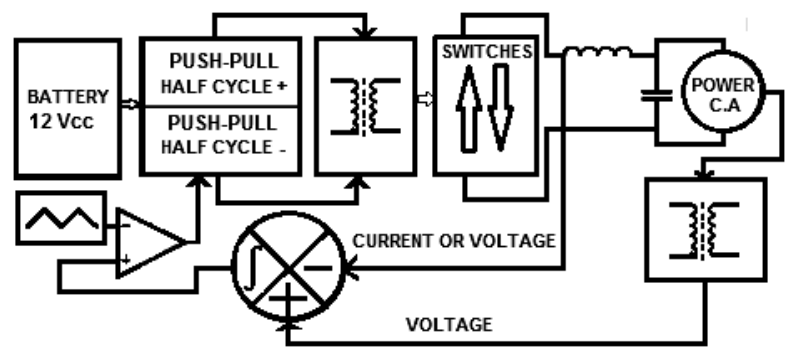

Fonte: Autor

\section{Circuito de potência e sequência de chaveamento.}

A forma de onda na saída do conversor devido ao chaveamento dos transistores é quadrada e modulada por largura de pulso de acordo com a tensão da rede elétrica. Apresentando todas as componentes de altas frequências. Como a finalidade do inversor é uma forma de onda senoidal na carga, foi desenvolvido um filtro LC passa baixas na saída do inversor para filtrar as componentes em altas frequências (BOYLESTAD; NASHELSKY, 2011), assim, a forma de onda na saída tem um comportamento senoidal. Para a sequência correta de acionamento das chaves do circuito de potência, os conversores devem seguir uma hierarquia em que cada conversor é acionado em um semi-ciclo, com uma sequência de chaveamento determinada pela carta de tempo, apresentada na figura 2. A sequência de funcionamento para o circuito de potência é descrita em seguida, com os oito passos de disparo das chaves para a formação do sinal alternado na saída do inversor. 
Figura 2 - Carta de tempo de acionamento das chaves.

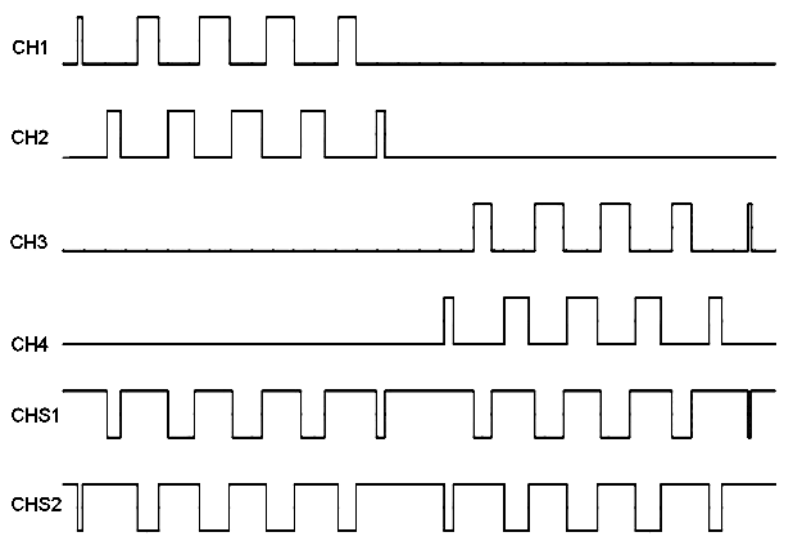

Fonte: Autor

O acionamento das chaves é dividido em duas partes com quatro passos cada. Os quatro primeiros passos são referentes ao semi-ciclo positivo, e os quatro passos subsequentes, ao semi-ciclo negativo. Devido às chaves do secundário estarem isoladas eletricamente das chaves do primário, foi necessário introduzir um atraso de tempo ajustável no acionamento das chaves do primário para que as condições de condução simultânea das chaves sejam ajustadas para um melhor sincronismo e diminuição de perdas por efeito joule, circuito esse que pode ser visualizado no anexo A. Os 8 passos são descritos a seguir, e o circuito elétrico de potência é apresentado no anexo B.

Passo 1: a chave $\mathrm{CH} 1$ conduz magnetizando o enrolamento LP2 e induzindo tensão nos enrolamentos secundários, para garantir o sentido da corrente na carga a chave CHS1 também deve conduzir e o sentido da corrente é apresentado na figura 3, levando-se em consideração as linhas em negrito e sentido da corrente nos diodos que conduzem nesse momento sendo que as demais chaves ficam abertas.
Figura 3 - Sentido de corrente no passo 1

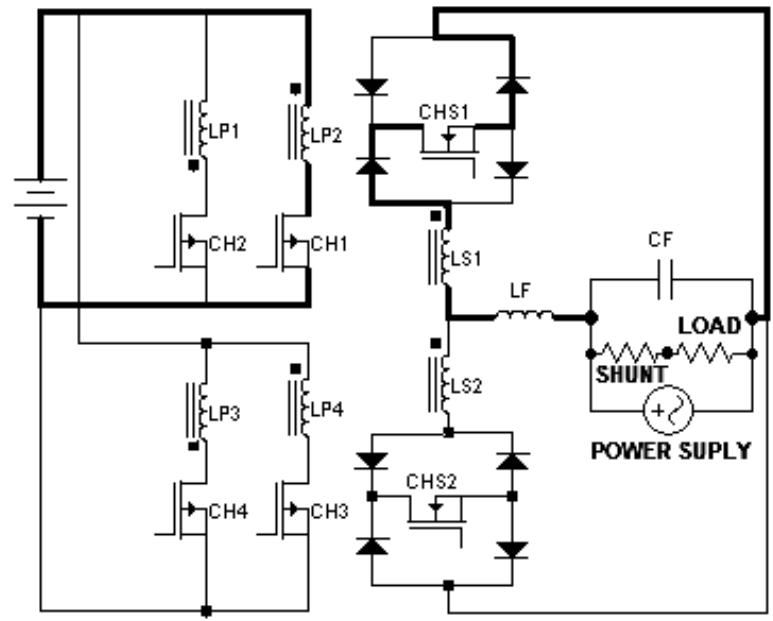

Fonte: Autor

Passo 2: a chave $\mathrm{CH} 1$ se abre e CHS2 entra em condução, isso equivale ao tempo morto entre as chaves $\mathrm{CH} 1$ e $\mathrm{CH} 2$, fazendo com que o fluxo de corrente entre em roda livre, e a tensão nos enrolamentos secundários seja nula, conforme a figura 4.

Figura 4 - Sentido de corrente no passo 2

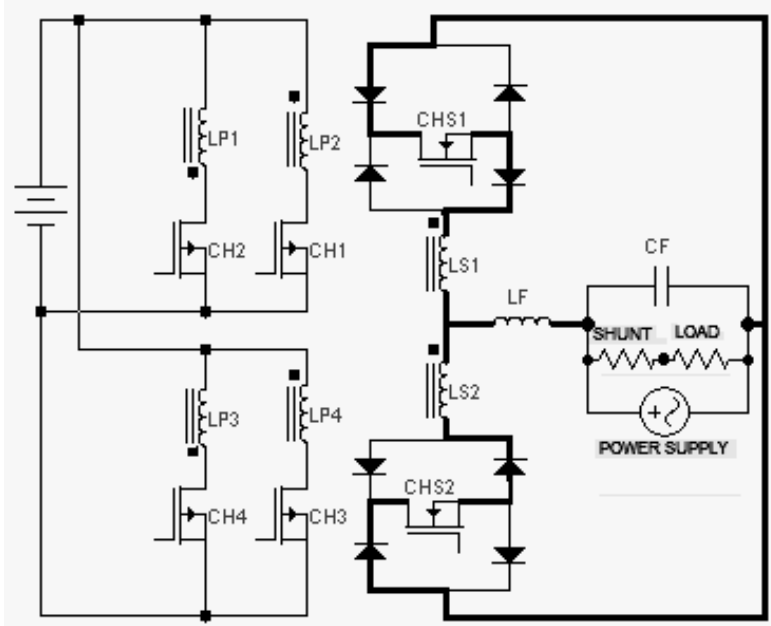

Fonte: Autor

Passo 3: a chave $\mathrm{CH} 2$ passa a conduzir induzindo tensão com polaridade oposta ao passo $1, \log o$ para manter o sentido da corrente na carga, CHS1 deixa de conduzir e CHS2 permanece conduzindo conforme mostra a figura 5 
Figura 5 - Sentido de corrente no passo 3

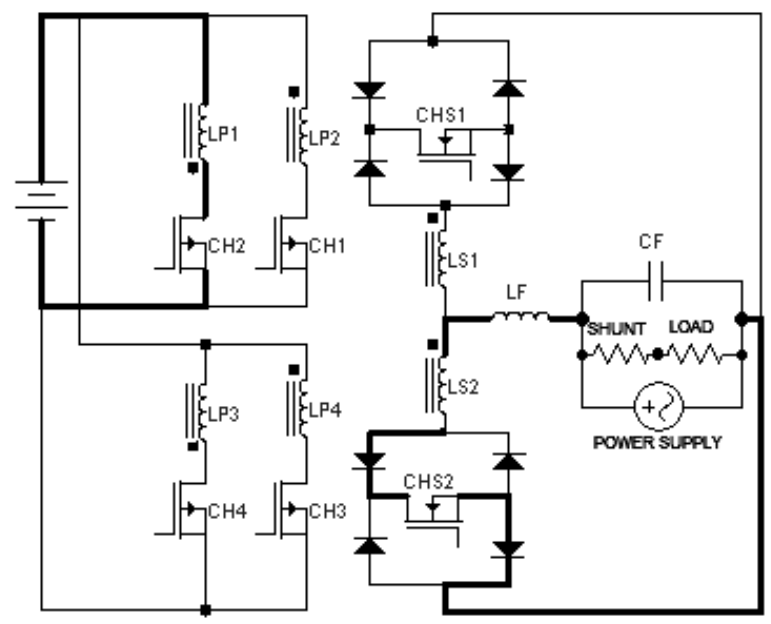

Fonte: Autor

Passo 4: é o tempo morto entre $\mathrm{CH} 1 \mathrm{E} \mathrm{CH} 2$, onde somente CHS1 e CHS2 conduzem, fazendo com que o fluxo de corrente entre em roda livre e a tensão nos enrolamentos secundários seja nula, conforme mostra a figura 6 .

Figura 6 - Sentido de corrente no passo 4



Fonte: Autor

Passo 5: a chave $\mathrm{CH} 3$ conduz magnetizando o enrolamento LP4 e induzindo tensão nos enrolamentos secundários, para garantir o sentido da corrente na carga a chave CHS2 também deve conduzir, conforme demonstra a figura 7.
Figura 7 - Sentido de corrente no passo 5



Fonte: Autor

Passo 6: a chave $\mathrm{CH} 3$ para de conduzir e CHS1 entra em condução, fazendo com que o fluxo de corrente entre em roda livre, conforme a figura 8.

Figura 8 - Sentido de corrente no passo 6

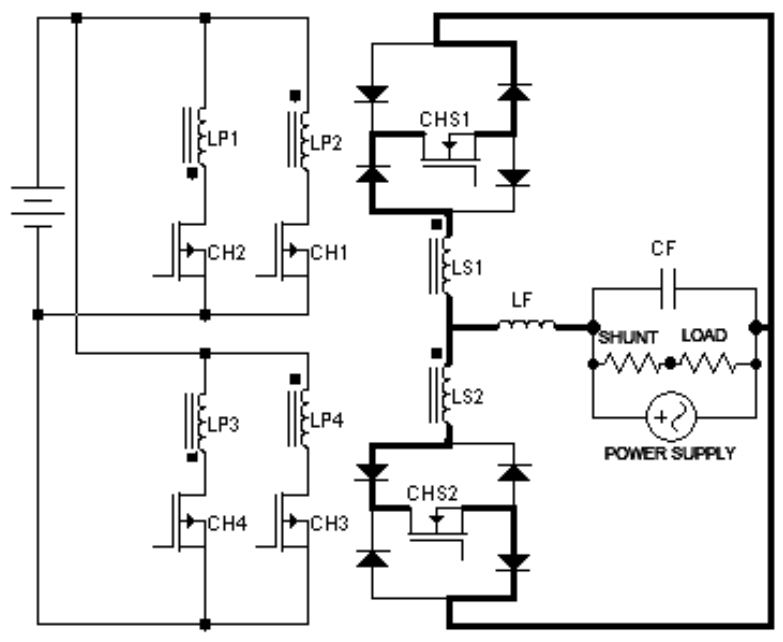

Fonte: Autor

Passo 7: A chave $\mathrm{CH} 4$ conduz induzindo uma tensão nos enrolamentos secundários com polaridade oposta ao passo anterior, para manter o sentido da corrente CHS2 deixa de conduzir e CHS1 passa a conduzir, conforme figura 9. 
Figura 9 - Sentido de corrente no passo 7

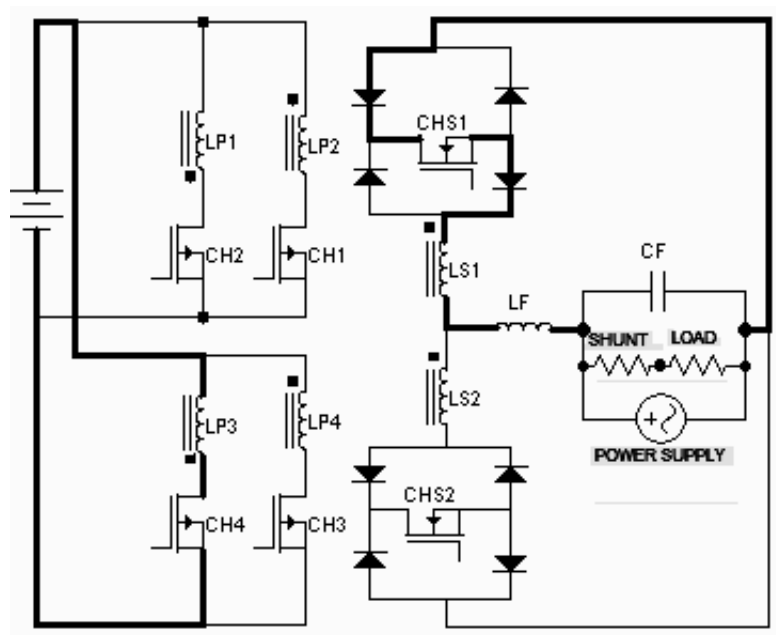

Fonte: Autor

Passo 8: A chave $\mathrm{CH} 3$ para de conduzir e temos um tempo morto de $\mathrm{CH} 3$ e $\mathrm{CH} 4$, enquanto $\mathrm{CHS} 1$ e CHS2 conduzem simultaneamente proporcionando o fluxo de corrente em roda livre conforme figura 10.

Figura 10 - Sentido de corrente no Passo 8

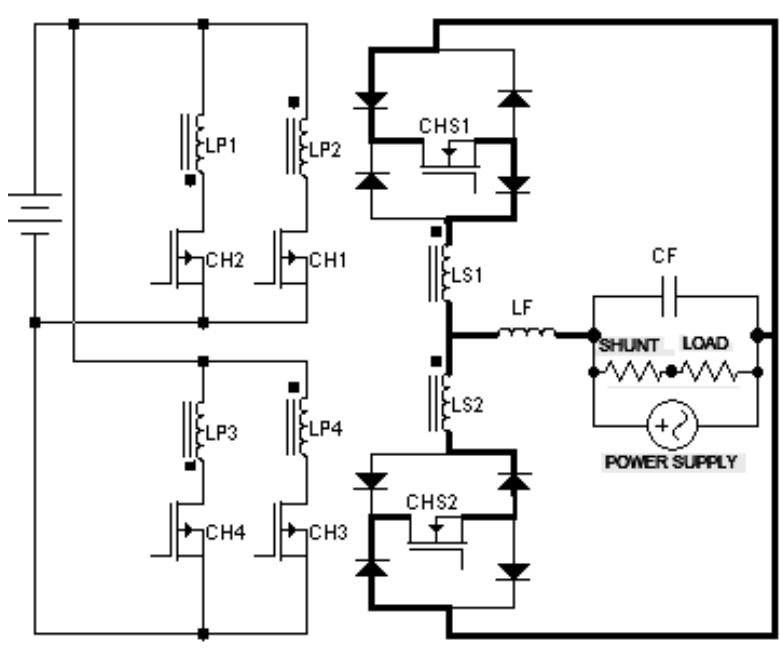

Fonte: Autor

Em paralelo com as chaves do circuito de potência foram inseridos circuitos snubbers (RASHID, 1999) diminuindo os picos de tensão sobre as mesmas devido ao chaveamento. Devido ao acoplamento ótico entre o circuito de acionamento e as chaves do secundário, foi necessário inserir uma constante de tempo variável para atrasar e assim sincronizar os sinais das chaves do primário em relação as chaves do secundário para diminuir o aquecimento devido a conduções não sincronizadas, o que pode ser visualizado no anexo A.

\section{Circuito de Comando}

$\mathrm{O}$ circuito de comando das chaves recebe a variação do sinal pwm (SEDRA- 2000) oriundo do circuito de controle para executar então as lógicas de acionamento e separação de sinais para as respectivas chaves, a lógica de acionamento é apresentada no diagrama da figura 11, e é desmembrado em seguida.

Figura 11 - Circuito de Comando

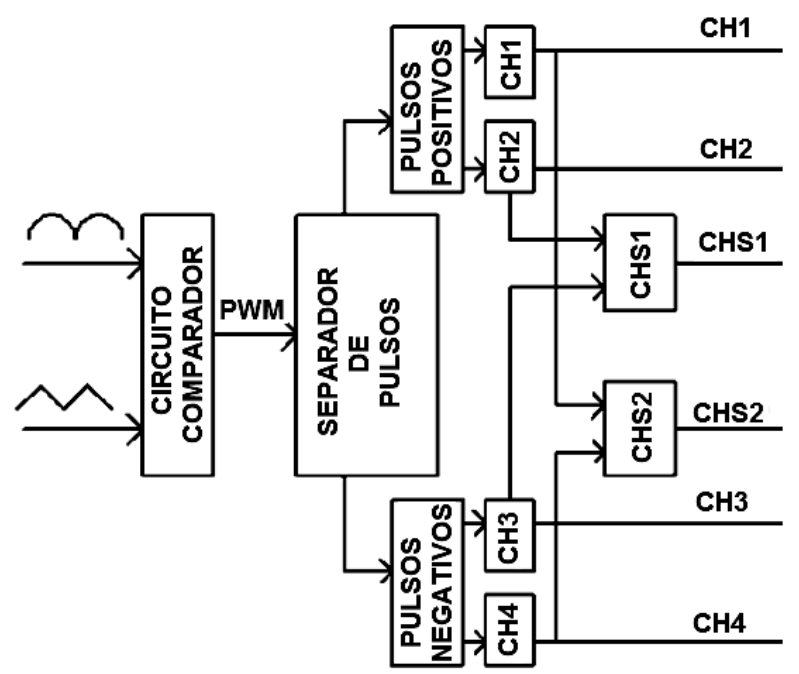

Fonte: Autor

Após a comparação do sinal retificado com a onda triangular, o sinal $p w m$ vai para o circuito separador de pulsos, este circuito faz a separação dos pulsos dos dois conversores, onde, quando o sinal da rede estiver no semi-ciclo positivo aciona as chaves do primeiro conversor, e quando estiver o semi-ciclo negativo aciona as chaves do segundo conversor, de acordo com a figura 12 . 
Figura 12 - Circuito separador de pulsos negativos e positivos.

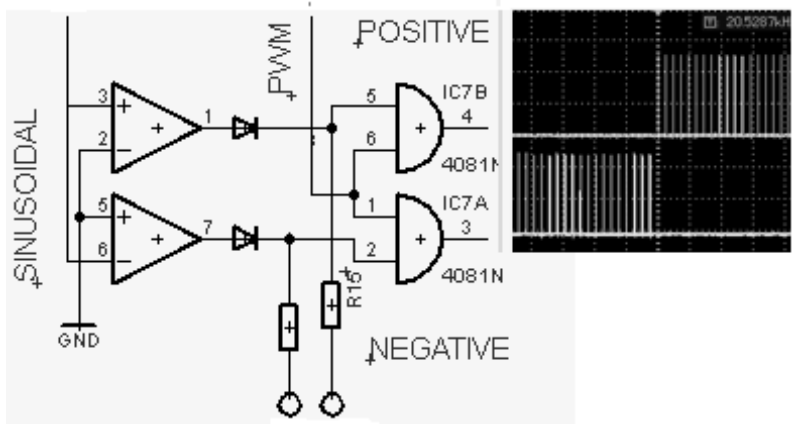

Fonte: Autor

Os pulsos positivos são então separados em dois novos pulsos para o conversor do semi-ciclo positivo, que acionarão as chaves $\mathrm{CH} 1$ e $\mathrm{CH} 2$, os pulsos referentes ao semi-ciclo negativo são separados em dois para acionamento do segundo conversor que fará o acionamento das chaves $\mathrm{CH} 3$ e $\mathrm{CH} 4$, a figura 13 apresenta o circuito e as formas de ondas.

Figura 13 - Circuito separador de pulsos das chaves do primário.

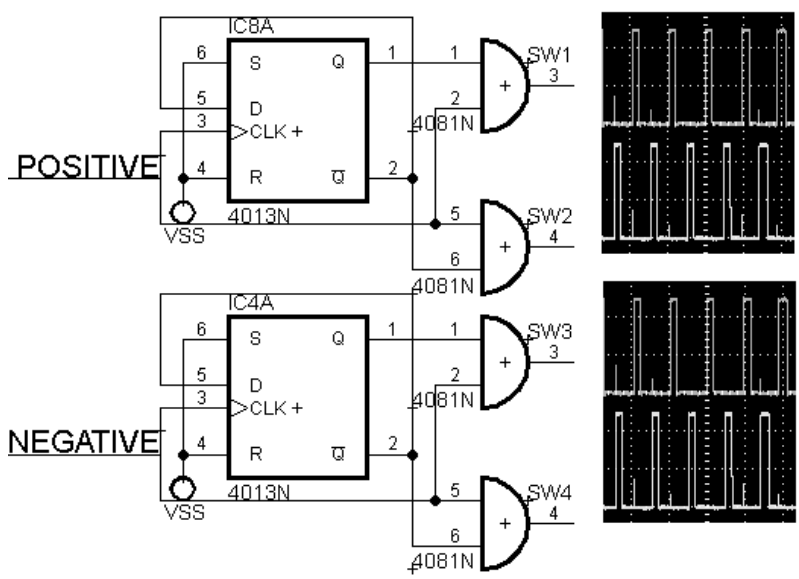

Fonte: Autor

Os pulsos das chaves dos enrolamentos do secundário que acionam as chaves CHS1 e CHS2 são formados pela lógica xnor entre os pulsos das chaves dos primários. As chaves do secundário (bidirecionais) são alimentadas por uma fonte de alimentação auxiliar que é chaveada e acionada pelo sinal proveniente do circuito gerador de ondas elaborado com o circuito integrado NE566 que fornece também uma saída com forma de onda quadrada. Para garantir a abertura das chaves bidirecionais, uma elevação de nível de tensão foi desenvolvida nas chaves para que o gate das mesmas trabalhem com referencial menor para garantir a abertura. As informações citadas nas linhas anteriores podem ser consultadas no anexo B que traz o circuito de potência completo. As formas de ondas nas chaves do secundário são apresentadas na figura 14.

Figura 14 - Circuito de pulsos das chaves do secundário

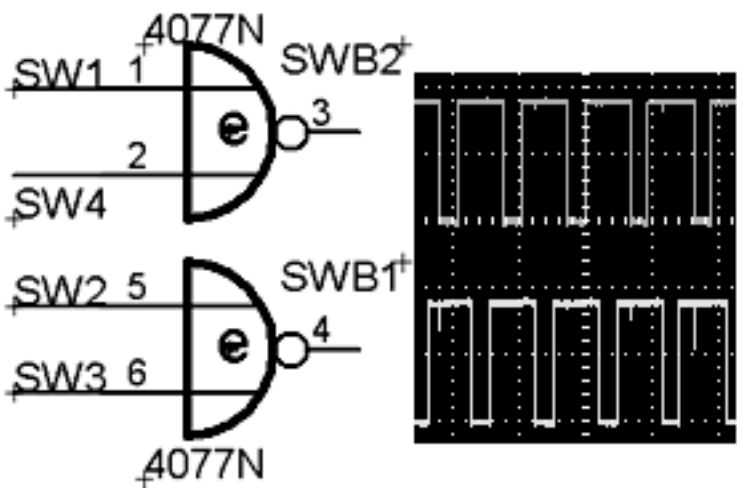

Fonte: Autor

\section{Circuito de controle}

Para controlar a tensão e a corrente na saída do inversor foi implementado um circuito de controle que faz o uso de uma ação integral para que se possa trabalhar com cargas variáveis e o mesmo possa compensar tal comportamento instável das cargas (OGATA-2003). O circuito de controle pode trabalhar de duas formas diferentes. A primeira forma é um controle com realimentação de tensão utilizando amostra da tensão de saída do inversor para a correção. Dessa maneira pode-se alimentar cargas não conectadas na rede elétrica tais como 
eletrodomésticos, lâmpadas, entre outros, mas que podem ser ligados ou desligados a qualquer momento. Logo o inversor necessita fazer a compensação de tensão na saída de forma a não fornecer variações de tensões às cargas. A segunda forma de controle diz respeito exatamente à injeção de potência em conjunto com a rede elétrica. Nessa maneira de trabalho, o inversor terá a saída conectada diretamente à rede elétrica, assim a tensão de saída do inversor não sofrerá alterações em caso de variação de carga devido à rede elétrica compensar. Mas, para o inversor injetar mais ou menos potência na rede devido a variação de carga, foi inserido um controle com realimentação de corrente, assim, quando a carga variar, a corrente da mesma seguirá esse comportamento, o controle irá perceber essa variação e fará a compensação injetando mais ou menos potência em forma de corrente. A figura 15 apresenta o circuito de controle, que pode trabalhar das duas formas citadas de acordo com a escolha da chave $\mathrm{S}$.

Figura 15 - Circuito de Controle

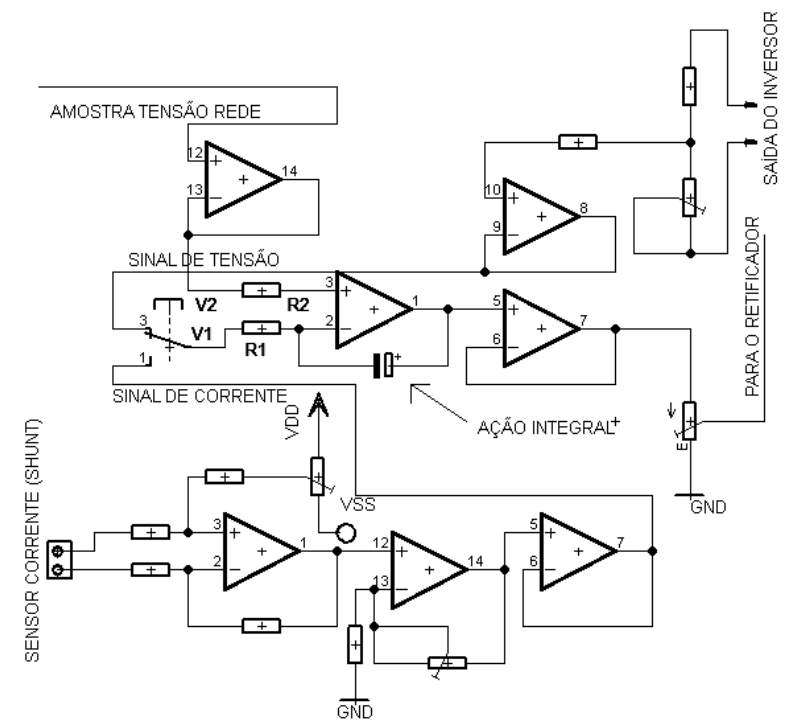

Fonte: Autor

\section{Dimensionamento do inversor}

Aqui é abordado o dimensionamento dos principais componentes do inversor para as seguintes características (MELLO, 2011):

$\left\{\begin{array}{l}\text { Potência de saída }: 100 \mathrm{~W} . \\ \text { Tensão de saída }: 127 \mathrm{~V}_{\mathrm{RMS}} \\ \text { Tensão de entrada }: 12 \mathrm{~V}_{\mathrm{CC}}\end{array}\right.$

Corrente rms na saída

$I_{R M S}=\frac{P_{O U T}}{V_{R M S}}=\left(\frac{100}{127}\right) / 2=0,394 \mathrm{~A}$

Corrente rms no primário de cada conversor

$I_{P}=\left(I_{S} \cdot D\right) \cdot N=0,394 \times 0,45 \times 25=4,432 \mathrm{~A}$

Onde: $\left\{\begin{array}{l}D=\text { razão cíclica } \\ \mathrm{N}=\text { relação de transformação } \\ \mathrm{Ip}=\text { corrente no primário } \\ \text { Is }=\text { corrente no secundário }\end{array}\right.$

Cálculo das chaves do primário

As chaves do primário devem suportar a tensão de o dobro de $V i$, ou seja: $V_{D S} \geq 24 V$

A Corrente $R M S$ no primário é de 4.432A, calculada anteriormente, para esse valores foram escolhidas para as chaves dos enrolamentos primários os transistores IRFZ48N que possuem as características elétricas mostradas a seguir.

\begin{tabular}{|l|l|}
\hline$V_{D S}$ & $55 \mathrm{~V}$ \\
\hline$I_{D}$ & $64 \mathrm{~A}$ \\
\hline Ptot & $140 \mathrm{~W}$ \\
\hline$R_{D S}($ on $)$ & $16 \mathrm{~m} \Omega$ \\
\hline
\end{tabular}

Cálculo das chaves do secundário

As chaves bidirecionais (CHS1 e CHS2) têm de suportar o dobro da tensão de saída, como a relação escolhida foi de 25 a tensão terá um valor de pico de: $2 \times 12 \times 25=600 \mathrm{Vcc}$, o que da margem em relação 
à modulação, e devem suportar também a corrente de saída máxima calculada anteriormente, assim foi escolhido os transistores FQB5N80 que possui as seguintes características.

\begin{tabular}{|l|l|}
\hline $\mathrm{V}_{\mathrm{DS}}$ & $800 \mathrm{~V}$ \\
\hline $\mathrm{ID}_{\mathrm{D}}$ & $4,8 \mathrm{~A}$ \\
\hline $\mathrm{P}_{\text {tot }}$ & $140 \mathrm{~W}$ \\
\hline $\mathrm{R}_{\mathrm{DS}(\text { on })}$ & $2,6 \Omega$ \\
\hline
\end{tabular}

Cálculo do transformador do conversor push-pull

Produto das áreas (Ap).

Adotando $\mathrm{B}=0.3, \mathrm{Ku}=0.1, \mathrm{Ps}=100 \mathrm{VA}, \mathrm{z}=1.136$, $\mathrm{f}=20 \mathrm{KHz}$ e $\mathrm{Kj}=397$ temos [2]:

$A_{P}=\left[\frac{7,96 \cdot P_{S} \cdot 10^{4}}{K j \cdot 0,3 x 40 \cdot 10^{3}}\right]^{z}=1,791 \mathrm{~cm}^{4}$

Utilizando um núcleo toroidal pode-se reduzir o volume para até $50 \%$ do núcleo não toroidal. O núcleo utilizado no projeto foi o modelo NT 53/32/20 devido à disponibilidade em laboratório, que comporta os enrolamentos primários e secundários, logo todos são acoplados magneticamente. O cálculo foi efetuado com frequência de $20 \mathrm{KHz}$, pois trabalha um conversor por vez, assim o gerador pwm irá trabalhar a $40 \mathrm{KHz}$.

Número de espiras do primário

$$
N_{P}=\frac{V_{i} \cdot D_{\max }}{A_{e} \cdot B \cdot f} \cong 5 \operatorname{esp}
$$

Área do cobre do enrolamento primário

$$
A_{c u}=\frac{I_{e f}}{J}=\frac{4.432}{258.18}=0.0102 \mathrm{~cm}^{2}
$$

Para diminuir o efeito skin (MELLO, 2011), foi utilizado 13 condutores de 28 AWG em paralelo o que resulta em uma área de cobre equivalente ao calculado na equação (5).

Número de espiras do secundário: Escolhendo uma relação de transformação de 25 temos

$N_{s}=N_{p} \times 25=125$ esp

Área do cobre do enrolamento secundário

$A_{c u}=\frac{I_{e f}}{J}=\frac{0.394}{258.18}=0.00153 \mathrm{~cm}^{2}$

Condutor 25 AWG.

Calculo do filtro LC de saída:

Adotando a freqüência de corte de $4 \mathrm{kHz}$.

$$
C_{f}=\frac{1}{4 \pi \cdot \xi \cdot f_{c} \cdot R_{0}}=138 n F
$$

Onde $\zeta=0,9$ é o fator de amortecimento escolhido de acordo com (AHMED, 2000). Adotando valor comercial de $220 \mathrm{nF}$ para o capacitor têm -se:

$$
L_{f}=4 \cdot R_{0}^{2} \cdot \xi^{2} \cdot C_{f}=18.24 m F
$$

\section{Resultados experimentais}

Os resultados experimentais apresentados a seguir foram divididos em cinco etapas. Na primeira etapa, os dados foram extraídos do inversor em malha aberta (BARBI; MARTINS, 2005), ou seja, apenas com uma carga resistiva, sem o fechamento de malha e sem conexão com a rede elétrica. A figura 16 apresenta a forma de onda na saída sem o filtro LC e com uma carga resistiva de $160 \Omega$ e utilizando para compor o sinal pwm somente uma amostra do sinal da rede elétrica. Verifica-se que o sinal apresenta as componentes em alta freqüência, devido ao acionamento das chaves. 
Figura 16 - Tensão na saída sem o filtro LC (malha aberta)

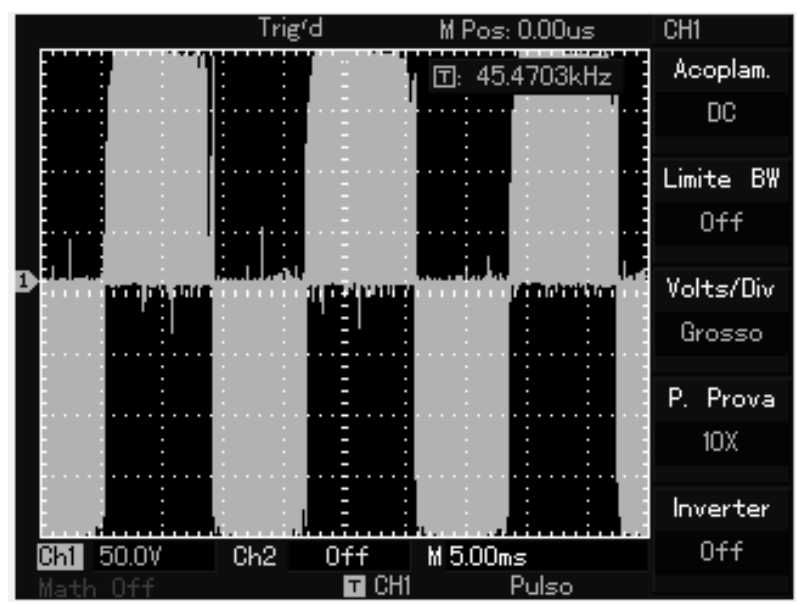

Fonte: Autor

$\mathrm{Na}$ segunda parte dos testes, foi adicionado o filtro LC na saída do inversor, assim foram eliminadas as freqüências acima da frequência de corte do filtro (1KHZ), resultando em uma forma de onda senoidal apresentada na figura 17.

Figura 17 - Forma de onda na saída com filtro LC (malha aberta)

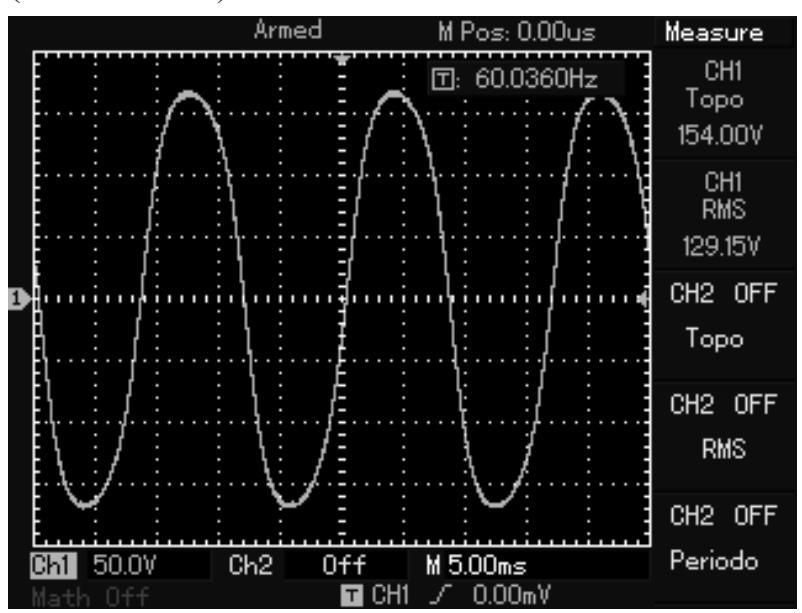

Fonte: Autor

Após testes em malha aberta, iniciou-se a terceira parte do experimento onde foi inserida a função integral entre a amostra da tensão da rede e uma amostra da tensão na saída do inversor, ainda sem ligar a saída do inversor à rede. Dessa maneira pode-se utilizar o inversor para alimentar cargas desconectadas da rede elétrica, e com a utilização da ação de controle, as cargas podem variar dentro das especificações de potência do inversor que o mesmo tenderá a manter a saída em torno de $127 \mathrm{Vca}$. A figura 18 apresenta a amostra do sinal da rede elétrica (canal 1- azul), e a amostra da tensão na saída do inversor (canal 2- amarelo), logo nessa maneira de funcionamento o sinal resultante da ação integral será uma senóide e seguirá a tensão da rede.

Figura 18 - Canal 1 amostra da tensão da rede

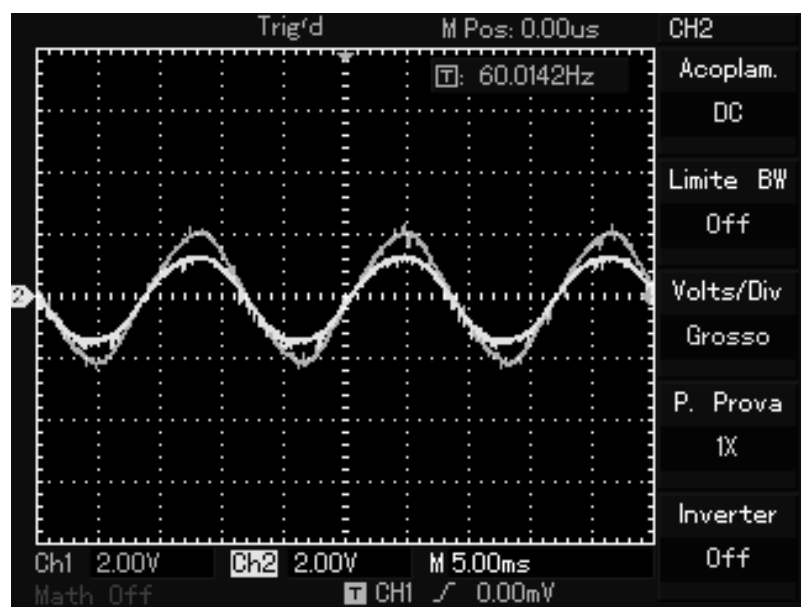

Fonte: Autor

$\mathrm{Na}$ quarta parte dos testes foi inserida uma realimentação em forma de corrente para compor o sinal de comparação para geração do pwm, uma amostra da corrente da carga retirada de um sensor shunt em série com a carga foi comparada com a amostra do sinal da rede elétrica. As formas de ondas da tensão na rede (canal 2- amarelo) e corrente na carga (canal 1- azul) podem ser visualizadas na figura 19, e a tensão resultante da ação integral será também uma senóide, mas agora seguirá a corrente da carga. 
Figura 19 - Canal 1 (amostra corrente na carga)



Fonte: Autor

O quinto modo de teste de funcionamento foi então conectar à rede elétrica a saída do inversor de modo que o mesmo possa fornecer potência à carga sincronizada com a rede elétrica, e dessa forma, quando a carga exigir mais potência, (diminuindo sua impedância), o inversor fará essa compensação em forma de corrente, já que a tensão de saída está fixada pela tensão de rede elétrica que está conectada à saída, assim o inversor injetará mais potência em forma de corrente para compensar a exigência da carga. Quando conectado à rede elétrica, a tensão na saída será fixada pela tensão da rede, logo a tensão da saída, e a da rede serão uma só, pode-se observar na figura 20. A tensão da saída já conectado a rede elétrica (canal-1 azul), e a corrente na carga (canal 2 - amarelo), assim, se a corrente aumentar, é porque a carga exige mais potencia, e o inversor injetará mais corrente na carga compensado pelo circuito de controle.
Figura 20 - Canal 1 (Tensão na saída conectada a rede elétrica)

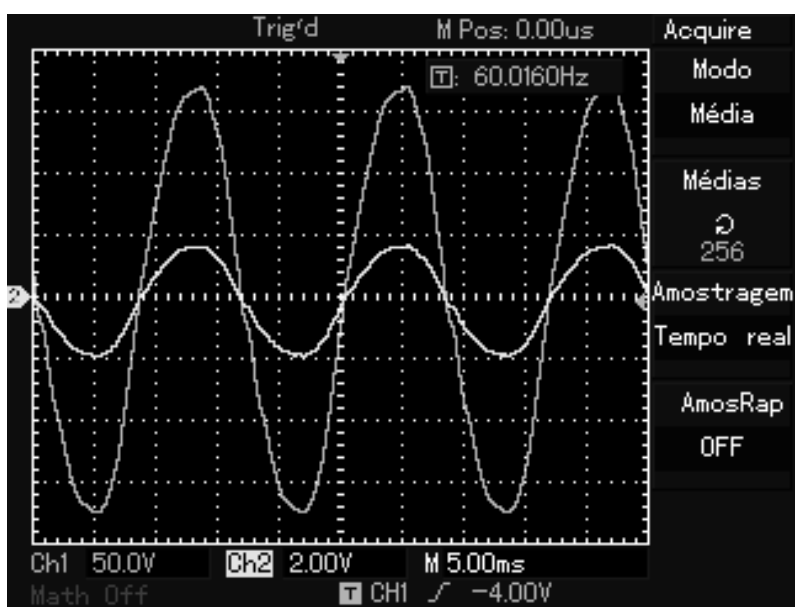

Fonte: Autor

\section{Conclusões}

O sistema desenvolvido mostrou-se compacto devido à utilização da técnica de chaveamento em alta frequência, conseguiu-se uma diminuição do peso e volume do núcleo do transformador, em conjunto com a escolha do núcleo modelo toroidal que possui melhores características de concentração das linhas de campo. Com um perfeito sincronismo entre as chaves do primário e secundário obtidos com o circuito de ajuste de tempo de atraso do disparo dos gates, pode-se diminuir as perdas por aquecimento provocado por possíveis conduções simultâneas. $\mathrm{O}$ rendimento conseguido foi aproximadamente $75 \%$ em função da potência escolhida para o projeto ser baixa (100W), pois somente com a alimentação dos circuitos de controle, fontes auxiliares e chaveamento é gasto aproximadamente $12 \mathrm{~W}$, mas, um projeto de maior potência fará com que o percentual de potência utilizada para alimentação dos circuitos seja menor em relação à potência total do projeto, proporcionando um melhor rendimento final. Os resultados experimentais validam a proposta do sistema de inversão que pode ser implementado em conjunto com qualquer sistema de co-geração 
de energia que possa fornecer tensões contínuas obtidas se possível de qualquer fonte renovável de energia.

Figura 21 - Protótipo do inversor

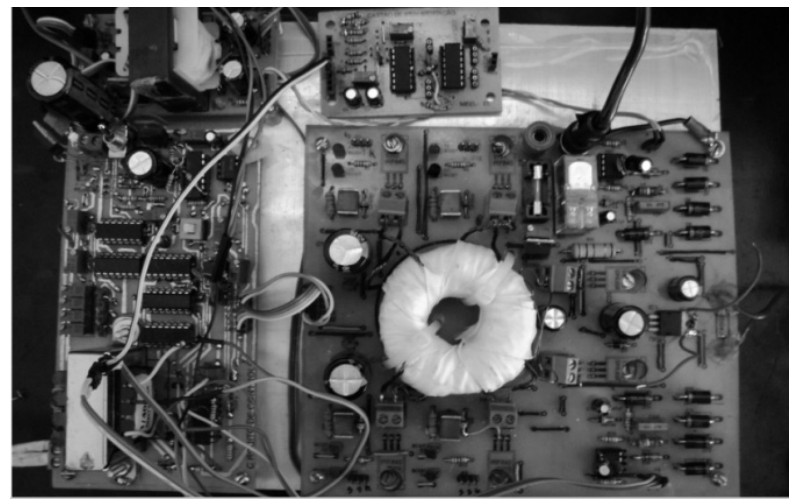

Fonte: Autor

\section{Referências}

AHMED, A. Eletrônica de potência. São Paulo: Prentice Hall, 2000.

BARBI, I. Projetos de fontes chaveadas.Florianópolis: Editora do autor, 2001.

BARBI, I.; MARTINS, D. C. Introdução ao estudo dos conversores $C C$-CA. Florianópolis: Edição dos Autores, 2005.

BOYLESTAD, R.; NASHELSKY, L. Dispositivos eletrônicos e teoria de circuitos. 8. ed. Rio de Janeiro: Peason Education do Brasil, 2011.

MELLO, L. F. P. Projeto de fontes chaveadas. São Paulo: Érica, 2011.

OGATA, K. Engenharia de controle moderno. 4. ed. São Paulo: Prentice Hall, 2003.

RASHID, M. H. Eletrônica de potência: circuitos, dispositivos e aplicações. São Paulo: Markron Books, 1999.

RODRIGUES, M.; TREVISO, C. H. G. New topology of voltage inverter based on push-pull converter with SPWM Modulator. In: CONGRESSO BRASILEIRO DE ELETRÔNICA DE POTÊNCIA, 2008. Proceedings. 2007.

SEDRA, A. S.; SMITH, K. C. Microeletrônica. 4. ed. São Paulo: Makron Books, 2000.
Recebido em 8 Fevereiro 2012- Received on February 8, 2012. Aceito em 13 Dezembro, 2012 - Accepted on December 13, 2012. 
ANEXO A

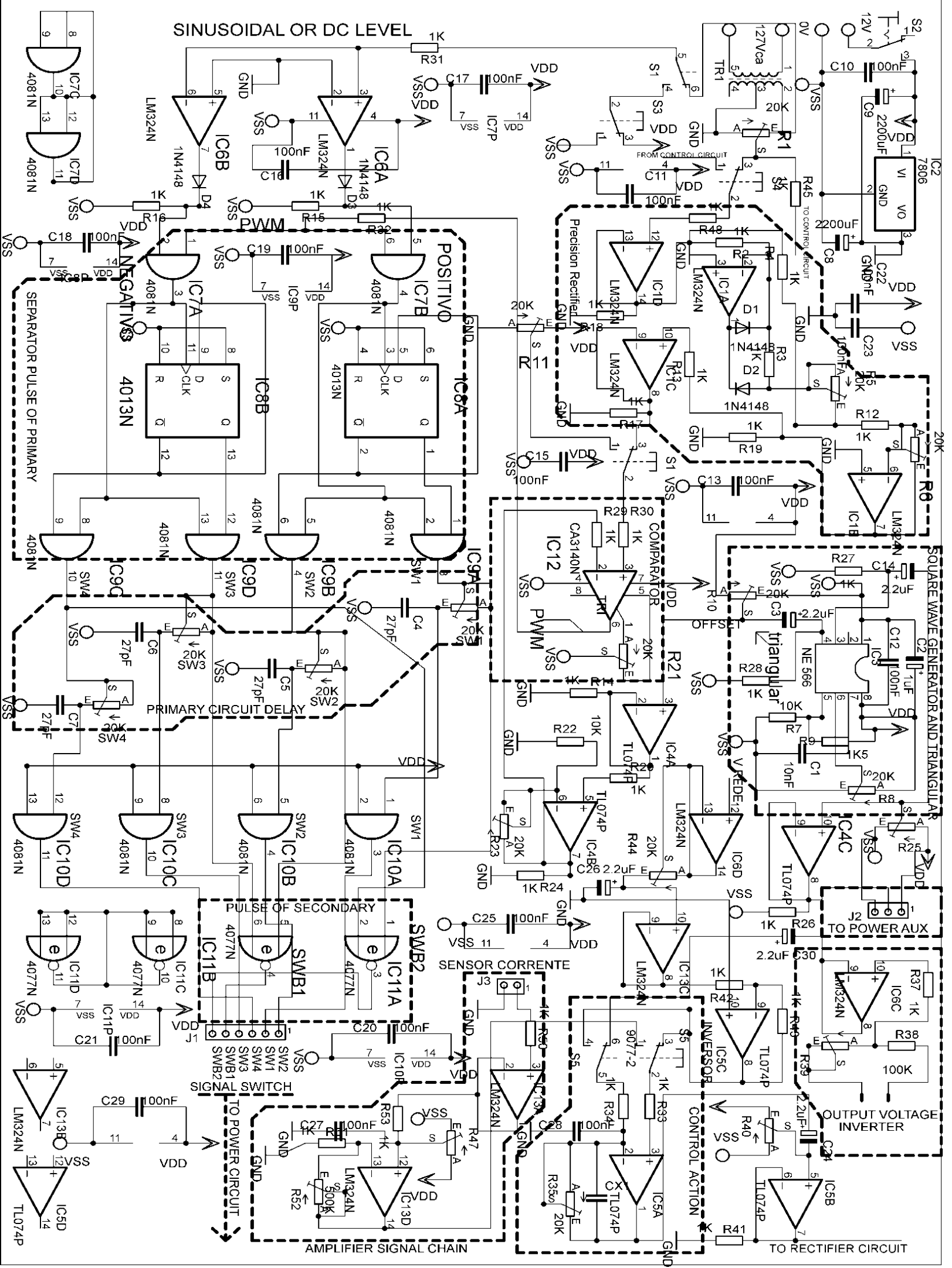




\section{ANEXO B}

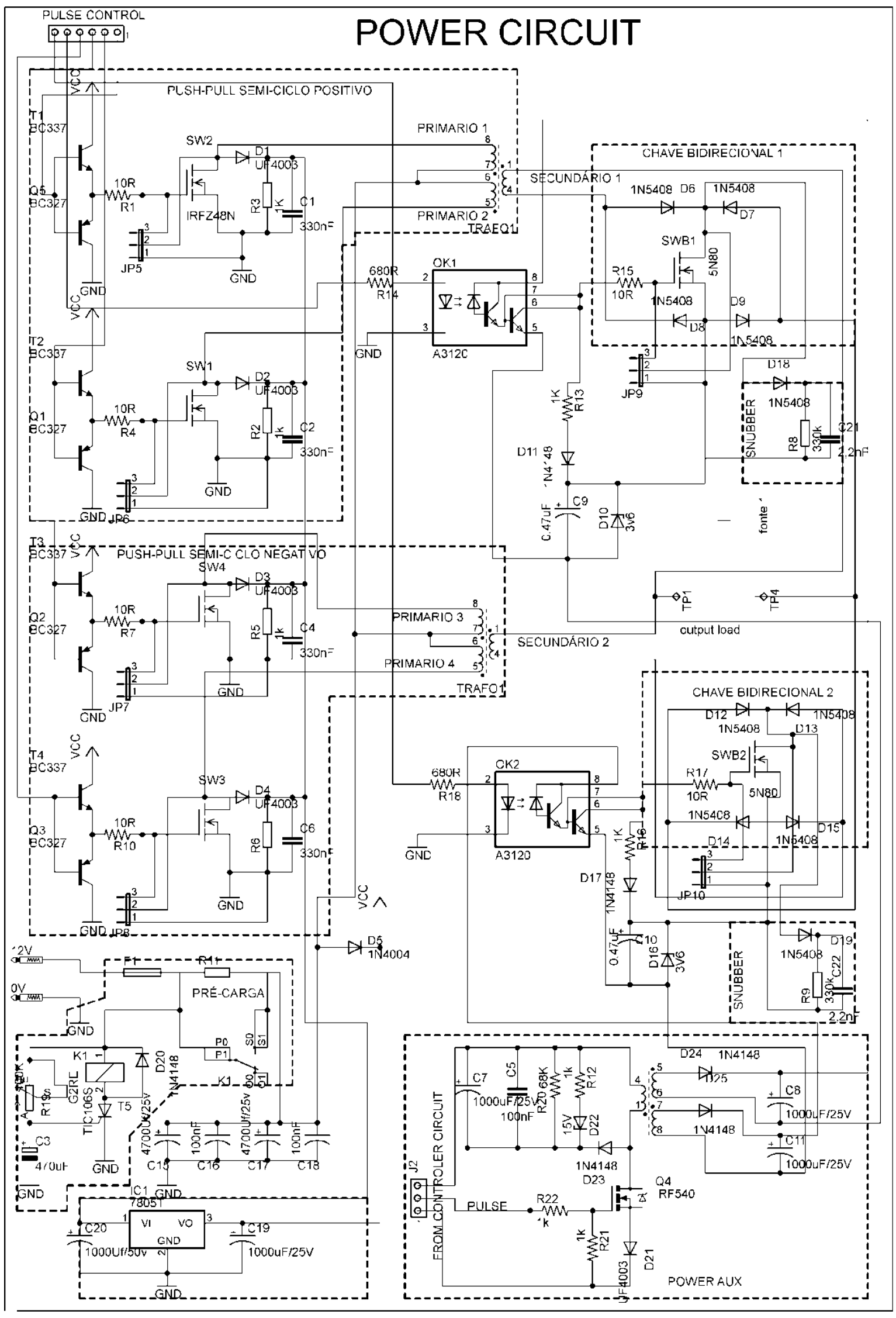

Semina: Ciências Exatas e Tecnológicas, Londrina, v. 34, n. 1, p. 31-44, jan./jul. 2013 
